\title{
De l'autostigmatisation aux origines du processus de stigma- tisation. A propos de l'enquête internationale « Santé mentale en population générale : images et réalités » en France et dans 17 pays
}

\author{
Jean Luc Roelandt*, Aude Caria, Imane Benradia y Simon Vasseur Bacle \\ Centre Collaborateur en Santé Mentale de l'Organisation Mondiale de la Santé (CCOMS, Lille) \\ (Received September 3, 2012; Accepted September 17, 2012)
}

RESUME: L'archétype du « fou » représente le paradigme du processus de stigmatisation et l'histoire de la folie apparaît comme une tentative de la faire disparaître en la médicalisant au profit de la notion de « maladie mentale ». Les résultats de l'enquête internationale SMPG nous montrent l'échec de la médicalisation de la folie à réduire la stigmatisation. Cette enquête décrit les représentations sociales associées aux archétypes du « fou », du « malade mental » et du « dépressif ». Réalisée en France (67 sites d'enquête) et dans 17 pays (20 sites internationaux), elle décrit les variants et invariants de ces trois archétypes.

Elle décrit les facteurs d'une stigmatisation importante pour le groupe « fou / malade mental » : non responsabilité, non contrôlabilité, médicalisation, mauvais pronostic et dangerosité. Quel que soit le pays, le noyau dur des représentations associant folie et danger est enraciné dans l'imaginaire collectif et le « malade mental » porte les attributs d'un « fou » médicalisé. A l'inverse, l'étiquette « dépressif » semble plus acceptable et moins exposée à la stigmatisation.

Très peu de personnes se reconnaissent dans la représentation collective du « fou » ou du "malade mental", même celles qui ont des troubles mentaux diagnostiqués. Dès lors, comment sortir de la dichotomie folie/raison, eux/nous à la base du processus de stigmatisation, si pour tout le monde, et même les personnes qui ont des troubles, le fou c'est l'autre? Ce sera peut-être le rôle des patients eux-mêmes de lutter contre la stigmatisation et l'auto stigmatisation dans les années à venir

Mots cles: stigmatisation, maladie mentale, représentations sociales, exclusion, dépression.

From the self-stigmatization to the origins of the stigmatization process. With regard to the survey "Mental health in the general population: images and realities» in France and 17 countries

\begin{abstract}
The archetype of the 'mad' represents the paradigm of the stigmatization process and the history of madness appear as an attempt to make it disappear by their medicalization for the benefit of the concept of 'mental illness '. The SMPG international survey results show the failure of the medicalization of madness to reduce stigma. This investigation describes the social representations associated to the archetypes of the "mad', the "mentally ill" and the "depressed".
\end{abstract}


Made in France (67 sites) and in 17 countries (20 international sites), she describes the variants and invariants of these three archetypes.

It describes the factors of significant stigmatization for the group ' $\operatorname{mad} /$ mentally ill ': non-responsibility, non-controllability, medicalization, poor prognosis and dangerousness. Regardless of the country, the hard core of representations combining madness and danger is rooted in the collective imagination and the "mentally ill" bears the attributes of a 'mad' medicalized. Conversely, the "depressive" label seems more acceptable and less exposed to stigma.

Very few people recognize themselves in the collective representation of the 'mad' or the 'mentally ill', even those who have diagnosed mental disorders. Therefore, how to exit to the dichotomies madness/reason, us /them that are at the base of the process of stigmatization, if for all the people, and even people who have mental disorders, mad it is the other? This may be the role of the patients themselves to combat the stigma and self-stigma in the years to come.

Keywords: stigmatization, mental illness, social representations, exclusion, depression.

\section{INTRODUCTION}

Selon Goffman (1975), la stigmatisation est un « processus dynamique d'évaluation qui discrédite significativement un individu aux yeux des autres ». L'archétype du « fou » représente le paradigme même du processus de la stigmatisation et de l'exclusion. Toute « l'Histoire de la Folie », depuis le Moyen Age, pourrait être écrite comme une tentative d'échapper à cette représentation stigmatisant, par glissements de sens, changements d'appellation et de concepts : du possédé par les démons, à l'usager des services de santé mentale, en passant par l'aliéné et le malade mental. Elle apparaît comme un processus de désacralisation de la « folie », pour tenter de la faire disparaître en la médicalisant au profit de la notion de « maladie mentale ».

Mais, les résultats de l'enquête internationale SMPG nous montrent l'échec de la médicalisation de la folie à réduire la stigmatisation (Roelandt J.-L., Caria A. et Defromont L., 2010). Plus récemment, on peut observer un nouveau glissement sémantique de la notion de "maladie mentale" vers celle plus globale, plus floue et plus acceptable de « dépression », comme une ultime tentative de camouflage ou de banalisation de la folie.

Aujourd'hui, si l'on parle facilement de « dépressif », de « déprimé », on observe également une tentative de dilution des appellations par l'utilisation de périphrases telles "personnes qui ont des problèmes de santé mentale", "personnes en souffrance psychosociale" ou "personnes vulnérables". Il s'agit de diluer le stigmate, de destigmatiser soi et les autres, de tenter de « changer de regard » pour considérer d'une manière positive les personnes qui jusqu'à présent faisaient peur, car renvoyant à l'archétype du « fou dangereux ». On peut comprendre cette tendance comme une tentative de retourner le stigma comme un gant, au profit des personnes qui le subissent. De transformer le "fou" d'agresseur potentiel en victime. 
Ces glissements de concepts et de sens sont interrogés par la recherche-action « La santé mentale en population générale : images et réalités », qui a décrit les représentations sociales associées aux trois archétypes $\mathrm{du}$ « fou », du «malade mental » et du « dépressif »'. Réalisée en France (67 sites d'enquête) et dans 17 pays (20 sites internationaux), elle permet l'analyse des variants et invariants dans les représentations liées à ces trois étiquettes.

\section{METHODOLOGIE}

Cette recherche-action internationale multicentrique, menée depuis 1997, a deux objectifs principaux :

1. Décrire les représentations sociales liées à la « folie », la « maladie mentale », la « dépression » et aux différents modes d'aide et de soins, et estimer la prévalence des principaux troubles psychiques dans la population générale âgée de plus de 18 ans.

2. Sensibiliser les partenaires sanitaires, sociaux, associatifs et politiques à l'importance des problèmes de santé mentale dans la population générale.

Pour chaque site participant à l'enquête, les données sont recueillies par des enquêteurs formés, grâce à des questionnaires administrés au cours d'entretiens en face à face auprès de personnes sollicitées dans la rue, anonymement, en respectant des quotas sociodémographiques (sexe, âge, catégorie socioprofessionnelle et niveau d'éducation) de manière à constituer un échantillon aussi représentatif que possible de la population vivant sur la zone géographique concernée. Pour chaque personne interrogée, les questions explorent ses propres représentations ainsi que la présence de troubles mentaux actuels ou passés et les recours thérapeutiques et/ou aides utilisés (Caria A., Roelandt J.-L. et Bellamy V., 2010).

Le Centre collaborateur de l'Organisation Mondiale de la Santé pour la recherche et la formation en santé mentale (CCOMS), dans le cadre de sa mission de collaboration internationale, fournit une aide méthodologique. L'enquête SMPG a ainsi été réalisée aux Comores, à Madagascar (2 sites), l'Île Maurice, Alger (Algérie), Bruxelles (Belgique), Halkida (Grèce), Nouakchott (Mauritanie), Tunis (Tunisie), Trieste (Italie), Séville (Espagne), Principauté des Asturies (Espagne) et Tanger (Maroc). Depuis 1997, l'enquête SMPG a été réalisée sur 79 sites : 66 sites nationaux et 13 internationaux, soit près de 70000 personnes interrogées : plus de 58000 individus en France (dont 2500 dans les DOM); plus de 11500 individus pour l'ensemble des sites internationaux.

L'hypothèse est qu'il y aurait un lien entre les représentations sociales, la stigmatisation, l'autostigmatisation et les modalités de prises en charge du « fou $», \mathrm{du}$ « malade mental » et du « dépressif ».

1 Les mots sont mis entre parenthèse car ils représentent des archétypes et non pas des diagnostics ni des réalités. 


\section{LES PRINCIPALES REPRESENTATIONS SOCIALES LIEES AUX ARCHE- TYPES «FOU », « MALADE MENTAL » ET « DEPRESSIF »}

1. Responsabilité, conscience, guérison

Quel que soit le site d'enquête, le « fou », le « malade mental » et le « dépressif» ne sont pas considérés par les personnes interrogées comme responsables de leur état (folie, maladie mentale ou dépression). Le « fou » et le « malade mental » ne sont pas non plus considérés conscients de leur état ni responsables de leurs actes, contrairement au « dépressif ». On voit donc bien que les représentations ne sont pas homogènes en santé mentale et varient selon les étiquettes et les diagnostics. On retrouve d'ailleurs ici la notion d'attributions causales (contrôlabilité et responsabilité) que Weiner (1988) décrit en lien avec les émotions et les attitudes, et donc la stigmatisation, liées à une étiquette donnée.

La perception de l'exclusion est retrouvée dans tous les sites d'enquête et s'échelonne selon trois gradients (du moins vers le plus) :

- la famille $<$ la société $<$ le monde du travail,

- « dépressif» $<$ « malade mental» $<$ « fou »

- $\quad$ sites de l'Océan indien $<$ du Maghreb $<$ Européens.

Les populations enquêtées pensent qu'il faut soigner contre leur gré un «fou », un « malade mental » et même un « dépressif » (ce dernier moins dans les sites européens). Ce qui est cohérent avec la perception de la non responsabilité de leurs actes et de leur état, ainsi que la perception de l'absence de conscience (ceci plus fréquemment dans les sites européens que dans les autres sites). Les populations des sites interrogés pensent majoritairement qu'un « fou » ou un « malade mental » ne peuvent guérir, contrairement au « dépressif ». Sauf à Madagascar où la population considère que tous les trois peuvent guérir, (en se soignant essentiellement avec des plantes et non pas avec des médicaments comme cela est pensé majoritairement ailleurs).

Guérir complètement semble quasiment impossible dans les différents pays pour le « fou » et le « malade mental», contrairement au « dépressif » (ex. : $80 \%$ des français pensent qu'un «dépressif» peut guérir complètement) Mais la guérison ne s'envisage pas comme possible sans aide (ex. : moins de 10\% des français pensent qu'un « fou » ou un « malade mental » peuvent guérir seuls, $30 \%$ un « dépressif »). 
Tableau 1. On Peut Guerir Completement

ON PEUT GUERIR COMPLETEMENT

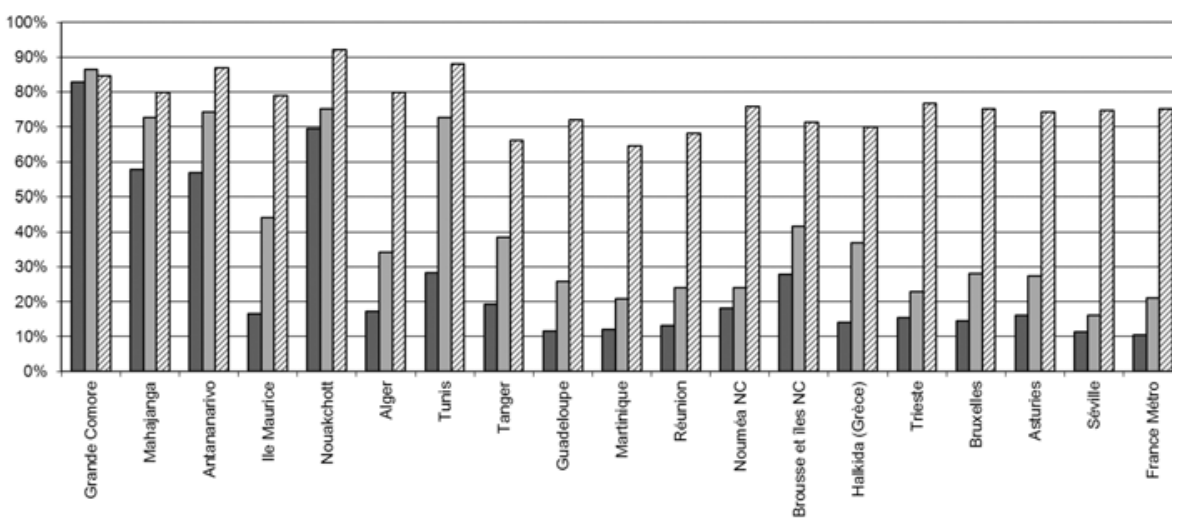

Source : Enquête SMPG, Ministère de la Santé-ASEP-CCOMS-EPSM LilleMétropole. Exploitation CCOMS

Hormis à Madagascar, 90\% des personnes interrogées dans les autres sites pensent qu'on ne peut soigner sans médicaments un « fou »ou un « malade mental », contre $60 \%$ pour un « dépressif.

Dans tous les sites, la population pense qu'un «fou» et un «malade mental» se reconnaissent d'abord à leurs comportements. Les actes tels que : « commettre un meurtre », « commettre un inceste », « commettre un viol », « être violent envers les autres », « battre régulièrement sa femme et ses enfants » sont attribués par 70 à $80 \%$ de l'échantillon français au « fou » et au «malade mental » mais très peu au « dépressif » (Tassone-Monchicourt C., Daumerie N. et Caria A., 2010). La même tendance est observable dans les autres sites, avec des nuances pour le meurtre qui n'est attribué à aucun des trois (ni «fou», ni «malade mental», «ni dépressif») à Trieste et à l'Ile Maurice, dans la majorité des cas.

\section{Les représentations des soins}

Concernant les soins, environ $30 \%$ des personnes interrogées accepteraient de soigner à domicile un proche « fou », $45 \%$ un proche « malade mental » et $80 \%$ un proche « dépressif». On observe ici un gradient dans la distance sociale exprimée, que l'on peut expliquer par les différentes associations (dangerosité, pronostic, ...) liées à chaque étiquette (Angermeyer \& Matschinger, 2003). Ces chiffres sont un peu plus élevés dans les sites de l'Océan Indien et à Nouakchott, identiques dans les autres sites. En France, on conseillera à un proche « fou » ou « malade mental » d'aller se faire soigner dans un hôpital psychiatrique (70\%), 
mais pas au proche « dépressif » (30\%). En France $40 \%$ des personnes interrogées déclarent être déjà entré dans un service de psychiatrie, 50\% à Nouakchott. Notons que c'est à Trieste, Séville et Lille-est, que les personnes interrogées conseilleraient le moins à un proche « fou », « malade mental » ou « dépressif» d'être soigné à l'hôpital psychiatrique, et le plus à être soigné en famille.

\section{Perception de soi-même}

La consommation déclarée de «médicament pour les nerfs 》 (somnifère, anxiolytique, antidépresseur, tranquillisant...) s'échelonne de $6 \%$ en Grande Comore à $37 \%$ en France et en Belgique. Le nombre de personnes déclarant avoir fait une psychothérapie varie de $0,8 \%$ à Antananarivo à $10 \%$ en France.

En France, 30\% des répondants déclarent connaître dans leur entourage un «fou», $40 \%$ un «malade mental» et $80 \%$ un « dépressif ». A Nouakchott, ces chiffres sont inversés : $60 \%-43 \%-40 \%$.

Tableau 2. Connaissance. Réponses a la Question : ¿Connaissez Vous Quelqu'un Qui Est Fou, Malade Mental Ou Dépressif?

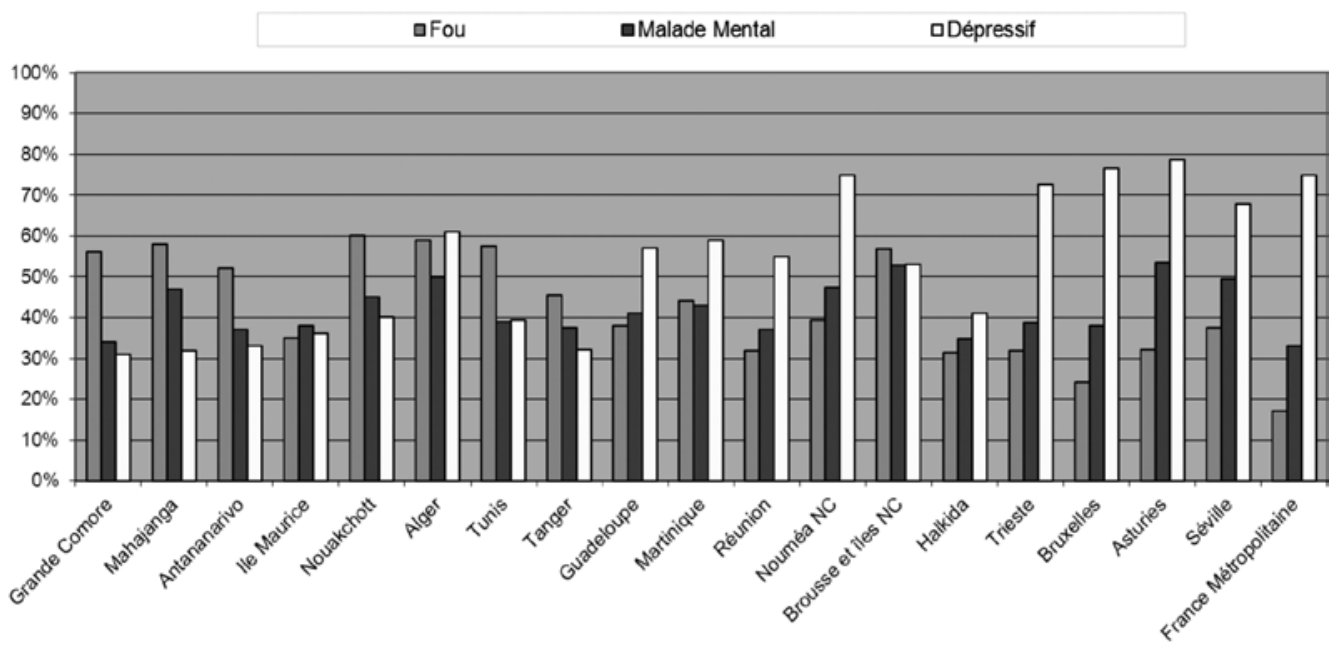

Source : Enquête SMPG, Ministère de la santé-ASEP-CCOMS-EPSM LilleMétropole. Exploitation CCOMS

Malgré la présence de troubles avérés par le questionnaire d'enquête et les soins déclarés par les enquêtés pour ces troubles (allant jusqu'à l'hospitalisation psychiatrique), quel que soit le site, très peu de personnes interrogées déclarent avoir déjà été soignées pour « folie » $(0,1-1 \%)$ ou « maladie mentale » $(0,7 \%$ $3,6 \%)$. Par contre, un nombre plus significatif déclare avoir déjà été soigné pour « dépression » (jusqu'à 19\% en France) 
Tableau 3. Expérience Personnelle. Réponses a la Question : «Avez-Vous Déjà Été Soigné-E Pour Folie ? Maladie Mentale ? Dépression?"

A DEJA ETE SOIGNE(E) POUR...

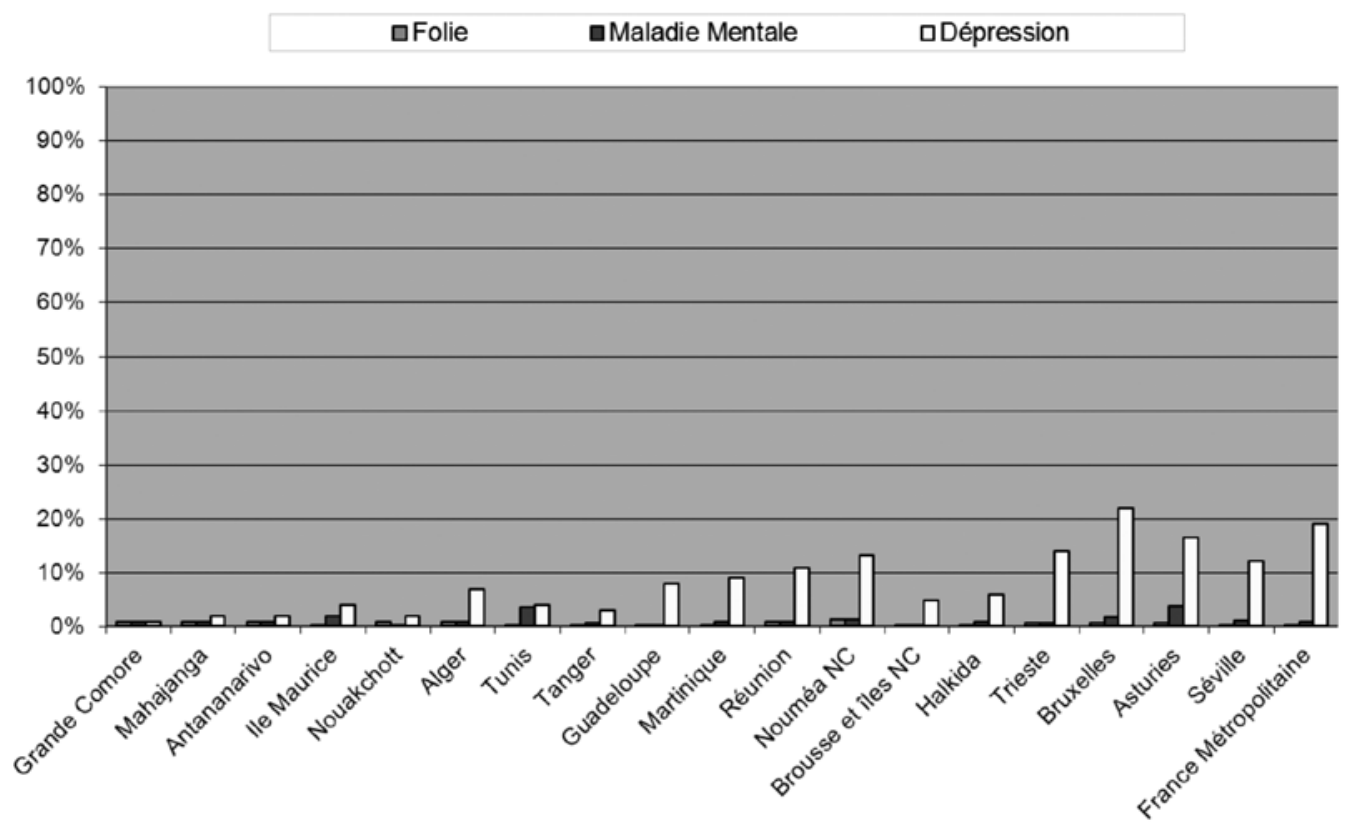

Source : Enquête SMPG, Ministère de la santé-ASEP-CCOMS-EPSM LilleMétropole. Exploitation CCOMS

Dans l'échantillon Nord/Pas de Calais ${ }^{2}$ (12 475 personnes), 1.24\% des répondants se considèrent comme «fou», $0.12 \%$ comme «malade mental» et $7.36 \%$ comme « dépressif $»^{3}$. Mais la moitié des répondants qui se considèrent « malade mental » ou « fou » n'ont aucun trouble psychique détecté par le questionnaire épidémiologique (Mini). Par contre $84 \%$ des personnes qui se déclarent comme « dépressives » présentent des troubles au Mini, mais ce n’est pas nécessairement un état dépressif qui est diagnostiqué.

Par ailleurs, $32 \%$ des répondants qui se considèrent ni « fou » ni « malade mental » ni « dépressif » présentent tout de même au moins un trouble au Mini (3 618 personnes sur 11 301). Et parmi les personnes identifiées par le questionnaire comme ayant un trouble (thymique, anxieux, psychotique, addictif), 75\% des répondants présentant ces troubles ne se considèrent ni « fou », ni « malade mental », ni « dépressif ».

Les répondants qui se considèrent comme « dépressif » déclarent beaucoup plus fréquemment de consommation de médicaments pour les nerfs et de suivi de psychothérapie que ceux qui se considèrent « fou » ou « malade mental ».

${ }^{2}$ Seul ce sous-échantillon régional permet les analyses suivantes

${ }^{3}$ Question : "Vous considérez-vous comme fou, malade mental, dépressif ou aucun des trois ?" 
Quelle que soit la pathologie identifiée par le questionnaire diagnostic, les enquêtés se considèrent majoritairement ni «fou», ni «malade mental», ni «dépressif».

Tableau 4. Variants et Invariants des Représentations

\begin{tabular}{|l|l|}
\hline \multicolumn{1}{|c|}{ Fou / Malade mental } & \multicolumn{1}{c|}{ Dépressif } \\
\hline Ce sont les autres & Ce peut être soi \\
\hline Considérés comme : & Considéré comme : \\
Non responsable de son état et de & $\begin{array}{l}\text { Responsable de son état et de ses } \\
\text { actes }\end{array}$ \\
ses actes & Ne devant pas être soigné contre \\
Devant être soigné contre son gré & son gré \\
(Médicaments) & Conscient de son état \\
Pas conscient de son état & Souffrant \\
Souffrant peu & Peu exclu \\
Exclu (sauf Madagascar) & Danger pour soi \\
Non guérissable (sauf Madagascar) & Suicide \\
Danger pour les autres & \\
Meurtre, viol, violence (Sauf & \\
Trieste et Ile Maurice) & \\
\hline
\end{tabular}

Source : Enquête SMPG, Ministère de la santé-ASEP-CCOMS-EPSM LilleMétropole. Exploitation CCOMS

Ce tableau illustre les cohérences internes des différents éléments constitutifs des trois archétypes étudiés. Deux grands groupes homogènes se dessinent : « fou » et «malade mental » d'un côté, « dépressif » de l'autre. Ces résultats éclairent sur le potentiel stigmatisant de ces étiquettes. On retrouve un ensemble de facteurs en faveur d'une stigmatisation importante pour le groupe « fou / malade mental » : non responsabilité, non contrôlabilité, médicalisation, mauvais pronostic et dangerosité. A l'inverse, l'étiquette « dépressif » semble moins exposée à la stigmatisation.

\section{DISCUSSION}

Comment désigner un stigmate qui en permanence se transforme et se travestit ? Le signe visible qui caractérise généralement le stigmatisé (marque physique, handicap, religion) est invisible en psychiatrie.

Dans les représentations sociales mises en évidence dans l'enquête SMPG, le « malade mental » porte les attributs d'un « fou » médicalisé. C'est un « fou » qui a le stigmate du handicapé mental, de la maladie génétique ou neurologique. L'archétype du « malade mental » s'est substitué historiquement à celui du 
« fou » avec l'essor de la médecine moderne qui a médicalisé la « folie » en lui proposant un soin.

Entré dans le champ de la médecine et de sa sémiologie, il doit pouvoir être reconnu, on cherchera donc à lui attribuer des stigmates physiques et médicaux. C'est en partie le rôle dévolu aux examens telles les coupes de cerveau à partir du $19 \mathrm{e}$ siècle, puis à l'imagerie médicale et la recherche génétique, qui cherchent elles aussi à identifier le signe du trouble. La marque qui est mise en évidence reste le plus souvent neurologique, et illustre, jusqu'à présent plus la déficience ou le dysfonctionnement que le trouble. Finalement tout se passe comme si pour accepter l'inacceptable, la folie, pour voir l'invisible marque, il fallait pouvoir le circonscrire et le designer, donc en trouver la trace tangible, dans le cerveau (la fameuse "pierre de la folie").

Mais là encore, pas de preuves tangibles sans ambigüités. Le fait de médicaliser le « fou » n'a pas fait disparaître les stéréotypes. Dans l'imaginaire collectif, la dangerosité attribuée au «malade mental » est dorénavant plus importante que celle attribuée au « fou ». La médicalisation de la "folie" n'a pas atteint le noyau dur de la représentation, soit l'association folie/danger devenue maladie mentale/danger. Les tentatives d'explication d'un trouble psychique uniquement sous l'angle « biologique » ne se semblent d'ailleurs pas fournir de résultats convaincants en termes de réduction de la stigmatisation (Angermeyer \& Schulze, 2001).

Pour la population, le "fou" et le «malade mental » sont essentiellement reconnaissables à leurs actes, c'est-à-dire aux troubles du comportement qui se déroulent dans la sphère privée et peuvent se répandre sur la place publique, associés alors avec la notion de danger (meurtre, viol et violences). Les actes dangereux fonctionnent donc dans l'inconscient collectif comme le signe visible de la "folie", en l'absence d'autres signes plus pathognomoniques (cf. plus haut). Pendant des siècles, la société a tenté de soigner la dangerosité en la rendant invisible, c'est-à-dire en excluant les fous et leur folie, hors la Cité, en les reléguant dans les asiles devenus hôpitaux psychiatriques, pour protéger les populations de leur dangerosité attribuée.

La séparation entre les autres (les « fous », les « malades mentaux ») et nous (les « dépressifs »), forgent le prototype de la ségrégation invisible c'est-à-dire non liée à des stigmates visibles (Rüsch, Angermeyer \& Corrigan, 2005). Les notions d'incurabilité, d'impossibilité de désigner visiblement et de danger potentiel associées aux archétypes du « fou » et du « malade mental », forgent la base d'une discrimination et d'une stigmatisation fortes.

Comment dans ces conditions pouvoir reconnaître les troubles mentaux lorsqu'ils nous concernent ? Quelle que soit la pathologie identifiée par le questionnaire diagnostic, les enquêtés se considèrent majoritairement ni «fou», ni «malade mental», ni «dépressif». Les répondants qui se considèrent comme « fou », « malade mental » ou « dépressif » ont les mêmes représentations concernant le «fou», le «malade mental» et le «dépressif» que le reste de la population. Le mécanisme de projection qui fait dire que le « fou », le « malade mental » c'est l'autre et pas soi, leur permet d'éviter un stigmate très fort, lié aux stéréotypes 
que tous partagent, du moins dans un premier temps. Par la suite, il faudra cacher la maladie si elle s'installe, et si on ne peut le faire, surtout quand le système de soins vous aura rejoint, tenter de la cacher, tenter de se faire soigner par d'autres personnes et en d'autres lieux qu'à l'hôpital psychiatrique, se dire avant tout «dépressif» ou trouver d'autres périphrases pour se désigner soi-même comme ayant à faire avec le champ de la psychiatrie. Cette volonté de dissimuler un diagnostic en santé mentale se retrouve dans de nombreux pays (Lasalvia et al., 2012, Thornicroft et al., 2009).

Ne pouvant mettre en évidence de stigmates biologiques, visuels ou sociaux de la « folie », ni de la « maladie mentale », la population a situé historiquement le « fou » à l'hôpital psychiatrique. C'est le lieu de projection fantasmatique, le lieu de la marque de la « folie » et de la « maladie mentale ». Dès lors, on comprend que l'on puisse plus facilement s'identifier au "dépressif", celui qui peut se soigner, guérir, à la maison ou dans des cliniques, mais pas à l'hôpital psychiatrique. L'étude internationale Indigo à d'ailleurs bien montré que pour les personnes ayant reçu un diagnostic de schizophrénie, le fait d'avoir été hospitalisé en psychiatrie est plus stigmatisant que le diagnostic de la maladie (Daumerie et al., 2012) De même, les patients qui sortent de l'hôpital diront avoir été soignés pour «dépression» et considèreront que les vrais « fous » sont ceux du pavillon d'en face ou de l'hôpital voisin.

\section{CONCLUSION : QUELQUES PISTES POUR CHANGER LES REPRESEN- TATIONS SOCIALES}

Les résultats de l'enquête SMPG nous montrent l'échec de la médicalisation de la folie à réduire la stigmatisation. Le noyau dur des représentations sociales associant folie et danger est enraciné dans l'imaginaire collectif. On peut observer un nouveau glissement sémantique de la notion de «maladie mentale» vers celle plus globale, plus floue et plus acceptable de « dépression », comme une récente tentative de gestion de la stigmatisation et un mécanisme d'adaptation à la société.

Très peu de personnes peuvent se reconnaître dans la représentation collective du « fou » ou du "malade mental", même celles qui ont des troubles mentaux diagnostiqués. Le "fou” ou le «malade mental», c'est l'autre, la stigmatisation reste trop forte et les mécanismes de défense de l'individu jouent pour éviter de se trouver confronté à ces notions quand il existe un trouble psychique. On ne s'étonnera pas que les troubles psychiques mis en évidence par les échelles diagnostiques soient avant tout attribués au malheur humain, aux événements de vie et que des stratégies d'évitement soient mises en œuvre par l'individu pour aller mieux. On comprend également que le premier recours déclaré pour les situations de mal-être psychique soit, dans presque tous les pays, l'entourage, la famille et le médecin généraliste, qui sera le premier pas vers la médicalisation du malaise diffus et invisible. L'absence de signes visibles attribuables aux troubles mentaux accentue la non reconnaissance de ces mêmes troubles. 
Et c'est quand l'examen clinique ne montrera rien de visible que l'orientation vers la psychiatrie sera proposée, si cela survient avant le trouble du comportement.

La médecine psychiatrique a permis de faire sortir les fous, devenus malades mentaux, des prisons, afin de les soigner. On le voit dans notre étude, les populations interrogées pensent légèrement plus que l'on peut soigner et guérir un « malade mental » qu'un « fou ». Mais la persistance de lieux de soins fermés maintient la confusion entre soin et enfermement, hôpital et prison, donc l'association folie-maladie mentale/danger. Cette association est en permanence entretenue par les médias et les politiques, qui ont les mêmes représentations que le reste de la population.

Dès lors, comment sortir de la dichotomie folie/raison, eux/nous, si pour tout le monde, et même les personnes qui ont des troubles mentaux, le fou c'est l'autre?

Si la médecine et la psychiatrie ont prouvé leur efficacité ces dernières décennies pour faire disparaitre, rendre invisible les symptômes, elle a aussi montré son impuissance à modifier les représentations stigmatisantes.

Ce sera peut-être le rôle des patients eux-mêmes de lutter contre la stigmatisation et l'auto stigmatisation dans les années à venir. Grâce aux concepts d'empowerment et de rétablissement, qui permettent à l'individu de reprendre le pouvoir d'agir sur sa maladie, de mener une vie socialement acceptable, de faire de sa maladie une chance, en prenant de la distance et en intégrant des connaissances sur ses troubles psychiques passés (WHO, 2010 ; Daumerie N., 2011). Le slogan des associations de patients nord américains «Nothing about us, without us» reste le leitmotiv de nombreuses associations de patients dans le monde. Cela sera possible aussi par la responsabilisation des individus qui ont des troubles psychiques comme citoyens, les troubles n'abolissant ni leurs droits ni leurs devoirs. Il conviendrait alors de favoriser leur témoignage, qu'il soit direct ou par média artistique interposé, afin de réfléchir collectivement aux questions liées à l'exclusion et à la stigmatisation en santé.

Ceci devra bien évidemment s'accompagner d'un changement dans les pratiques de soins, une modification de la place de l'hospitalisation psychiatrique dans l'offre de soins, le moins possible et le plus court possible, en services ouverts. Ces changements devront s'appuyer sur une modification profonde des cursus de formation initiale et continue, intégrant la conception du soin basé sur le rétablissement et l'empowerment. Les représentations sociales basculeront, quand les troubles ne seront plus assimilés à des comportements dangereux, quand le rétablissement ou la compensation prévaudront sur la conception d'incurabilité, quand les pratiques psychiatriques changeront et quand les mots pour désigner les troubles deviendront les plus anodins possibles, afin de permettre à la population de s'y identifier sans peur.

Tout cela finira peut-être par modifier les comportements et attitudes, comme cela est apparent dans les résultats des sites de Trieste, Séville et Lille-est, soit les sites ayant développé fortement les soins dans la communauté (Quidu \& Es- 
caffre, 2010).

Mais il conviendra de garder à l'esprit que nous sommes tous concernés par les représentations sociales et les stéréotypes, et par les mécanismes psychiques que nous mettons en place pour limiter les effets de la stigmatisation. En particulier, personne n'échappe à la distinction faite entre «eux» - les «fous», «malades mentaux», et toutes les autres appellations médicales ou supposées non stigmatisantes- et «nous» - les autres- qui fonctionne pour tous, «sains d'esprit» et soidisant «malades». Le slogan «Rien à propos de nous sans nous « des associations d'usagers nécessite donc que le nous englobe le «eux» aussi, afin d`éviter le clivage à l'origine de la stigmatisation. Donc un changement complet de paradigme pour les années à venir.

\section{BIBLIOGRAPHIE}

Angermeyer, M. C., Matschinger, H. (2003). The stigma of mental illness: effects of labelling on public attitudes towards people with mental disorder. Acta Psychiatrica Scandinavica, 108, 304-309.

Angermeyer, M. C., Schulze, B. (2001) Reducing the Stigma of Schizophrenia: Understanding the Process and Options for Interventions. Epidemiologia e Psichiatria Sociale, 10, 1-5

Caria, A., Roelandt, J. L., Bellamy, V. et al. (2010). Santé mentale en population générale : images et réalités (SMPG). Présentation de la méthodologie d'enquête. L'Encéphale, Supplément au n³, 1-6

Daumerie, N., Vasseur-Bacle, S., Giordana, J. Y, Roelandt, J. L, BourdaisMannone, C. (2012) La discrimination vécue par les personnes ayant reçu un diagnostic de troubles schizophréniques. Premiers résultats français de l'étude INDIGO. Encéphale, 38, 224-231.

Daumerie, N. (2011), L'empowerment en santé mentale : recommandations, définitions, indicateurs et exemples de bonnes pratiques. Santé de l'homme $\mathrm{n}^{\circ} 413$, mai-juin, 8-10

Goffman, E. (1975). Stigmate, les usages sociaux des handicaps. Paris, Editions de Minuit.

Quidu, F. \& Escaffre, J. P. (2010) Les représentations du «fou» du «malade mental», du «dépressif» et les opinions vis-à-vis des hôpitaux psychiatriques sontelles homogènes selon les populations des sites enquêtés ? L〉Encéphale, Volume 36, Issue 3, Supplément 1, Pages 15-19.

Roelandt, J. L., Caria, A., Defromont, et al. (2010) Représentations du fou, du malade mental et du dépressif en France. L'Encéphale, Supplément 1 au n³, 7-13.

Rüsch, N., Angermeyer, M. C., Corrigan, P. W. (2005) Mental illness stigma: Concepts, consequences, and initiatives to reduce stigma. European Psychiatry, 20, 529-539.

Tassone-Monchicourt, C., Daumerie, N., Caria, A. et al (2010) Etats dangereux et troubles psychiques : images et réalités. L'Encéphale, Supplément 1 au 
$n^{\circ} 3,21-25$.

Thornicroft, G., Brohan, E., Rose, D., Sartorius, N. (2009). The INDIGO Study Group. Global Pattern of Anticipated and Experienced Discrimination against People with Schizophrenia. The Lancet, 373, 408-415

Weiner, B., Perry, R. P. \& Magnusson, J. (1988). An attributional Analysis of Reactions to Stigmas. Journal of Personality and Social Psychology, 55, 738748.

WHO (2010). User empowerment in mental health - a statement. Copenhagen, WHO Regional Office for Europe. 